\title{
A INFLUÊNCIA DA ELEVAÇÃO RELATIVA DO TERRENO SOBRE A INFESTAÇÃO LARVÁRIA PELO MOSQUITO AEDES AEGYPTI
}

\author{
Diego de Sousa Ribeiro Fonseca \\ Universidade Federal de Minas Gerais/Instituto de Geociências (UFMG/IGC) \\ diegosousarf@gmail.com \\ Ricardo Alexandrino Garcia \\ Universidade Federal de Minas Gerais/Instituto de Geociências (UFMG/IGC) \\ alexandrinogarcia@gmail.com
}

\begin{abstract}
RESUMO
O objetivo do trabalho foi examinar a correspondência dos focos do mosquito Aedes aegypti com dados de altitude e declividade do ambiente citadino de Montes Claros-MG. A partir dos resultados, o assunto poderá contribuir nessa temática necessária, a respeito da qualidade urbana e a geografia da saúde social. A metodologia consistiu na aquisição, organização e espacialização dos dados sobre os focos positivos do Aedes aegypti; manipulação do modelo digital de elevação Topodata; aplicação do Índice de Moran; e teste da correspondência associativa entre as variáveis, por meio da regressão linear múltipla. Os resultados mostraram um coeficiente de determinação $\left(R^{2}\right)$ final igual a 0,973 - o que denotou alto poder preditivo para a combinação das variáveis independentes trabalha das. O Índice Breteau de 2016 foi aquele com maior peso explicativo $(p<0,01)$ entre o conjunto de dados para a explicação da infestação larvária ocorrida em 2017 . Foi verificado que a altitude média foi significativa nessa associação $(p<0,05)$, e apresentou correlação negativa conforme o coeficiente parcial de regressão $(-6,460$ e $t=-2,157)$. Em segundo plano, a altitude máxima foi significativa $(p<0,08)$. Chegou-se a conclusão de que, numa relação do período anterior com o seguinte, nas áreas de maior incidência larvária, qua nto mais baixa é a altitude, maior é a infestação pelo mosquito.
\end{abstract}

Palavras-chave: Aedes aegypti. altitude. Regressão Linear Múltipla. Planejamento das cidades. Geografia da Saúde.

\section{THE INFLUENCE OF TERRAIN SLOPE ELEVATION ON LARVAL INFESTATION BY THE AEDES AEGYPTI MOSQUITO}

\begin{abstract}
The objective of this work was to examine the relationship between Aedes aegypti mosquito neighbourhood presence with databases of slope elevation and environmental declivity in Montes Claros, Minas Gerais. Knowledge of this relationship has implications for urban quality and social health geography. The study methodology consisted of: the acquisition, organisation and spatialisation of databases for positive the presence of Aedes aegypti; the manipulation of digital modeling elevation data from Topodata; the application of Moran's Index; and an evaluation of the associative correspondence between the variables, using a multiple linear regression model. The coefficient of determination $\left(R^{2}\right)$ was 0.973 , indicating a high predictive power for the combination of variables. The Breteau Index from 2016 had the best explicative power $(p<0.05)$ among the set of databases to explain the larval infestation that occurred in 2017 . Terrain elevation had a significant association $(p<0.05)$ with infestation, and was negatively correlated with the partial regression coefficient $(-6.460$ e $t=-2.157)$. In the second model, the maximum elevation was significant $(p<0.08)$. As such, results suggest that in a relation of the previous period with the following one, in the areas of greater larva incidence, the lower the mean slope elevation, the larger the mosquito infestation.
\end{abstract}

Keywords: Aedes aegypti. Slope elevation. Multiple Linear Regression. Urban planning. Health of Geography.

\section{INTRODUÇÃO}

Problemas se tornam irresolúveis enquanto posições unilaterais firmes se sobrepõem a realidade ampla e complexa dos fatores envolventes. Os dilemas urbanos atuais são consequência das decisões pautadas

$\begin{array}{llllll}\text { Caminhos de Geografia } & \text { Uberlândia-MG } & \text { v. 22, n. 82 } & \text { ago./2021 } & \text { p.13-27 } & \text { Página } 13\end{array}$


apenas nos aspectos econômicos do capital financeiro intrincado. Perspectivas futuras são pessimistas quanto à qualidade do ambiente habitado, caso somente o interesse monetário venha prevalecendo.

A respeito do ambiente urbano, são notados em várias cidades do Brasil, de tempos em tempos, o surgimento e o ressurgimento de vetores de doenças, tal como o mosquito Aedes aegypti, o qual, à medida que ocorre o crescimento urbano, a infestação larvária parece aumentar, af etando toda a sociedade. Tal inseto é o vetor de transmissão de enfermidades como a dengue, febre amarela, febre chikungunya e o zika vírus (ALMEIDA et al., 2009).

Somado ao assuntado, é sabido que, atualmente, inexiste vacina eficaz a todos os sorotipos circulantes transmitidos pelo Aedes aegypti, a não ser para a febre amarela, tornando a melhor opção o co ntrole da praga. Tal atividade tem sido realizada por meio da eliminação dos criadouros potenciais, através da aplicação de larvicidas e inseticidas nos depósitos de água a fim de combater as formas adultas que estão no perío do de transmissibilidade. O combate eficiente está respaldado numa ação combinada de fatores envolvendo: o ser humano, o vírus, o vetor e o ambiente (KONISHI, 2011; GUPTA; REDDY, 2013; MESSINA et al., 2015; RIZZI et al., 2017).

O Brasil tem detectado surtos da dengue desde o século XIX. Epidemias ocorridas no Rio de Janeiro em 1846; no estado de São Paulo em 1852 e 1916; e em Niterói no ano 1923; são exemplos relatados na literatura (PINHEIRO; CORBER, 1997; NEVES, 2005). A doença hoje conhecida como dengue, já foi chamada por outros nomes populares, tais como: polca, patuleia, urucubaca, febre eruptiforme, febre quebra-ossos. Entre 1920 e 1980, houve certo esforço epidemiológico para combater a dengue nos países latino-americanos. O Brasil, inclusive, foi pioneiro na tentativa da eliminação sistemática do mosquito Aedes aegypti, especificamente no ano de 1955, quando realizou junto com outros países vizinhos, campanha internacional objetivando a eliminação do mosquito, promovida pela Superintendência de Campanhas de Saúde Pública (SUCAM) e o Departamento Nacional de Endemias Rurais (DENERu), ambos vinculados ao Ministério da Saúde. No entanto, em seguida aconteceram as primeiras negligências nos programas de controle do $A$. aegypti, desde o governo Juscelino Kubitschek aos governo s militares, quando a moléstia se alastrou pelos estados brasileiros (CONSOLI; OLIVEIRA, 1994; FORATINI, 2002).

O reconhecimento de áreas com maior propensão à inf estação larvária do vetor pode ser mais incisivo quando a intenção é chegar aos locais prioritários para ações de combate aos problemas na saúde coletiva (MORENO; BARATA, 2012). Em tal zoneamento, a contextualização dos distúrbios ep idêmicos, associando-os às características dos espaços geográficos aos quais determinadas populações habitam, é fundamental para o entendimento, com maior abrangência dos processos espaciais dinâmicos dessas anomalias (SAWYER et al., 2002).

O objetivo deste estudo é examinar a correspondência dos focos do mosquito Aedes aegypti com a altitude e declividade do ambiente citadino de Montes Claros-MG. O prag matismo envolvendo tais fatores está na metrificação do poder dessa associação, a fim de que seja possível, em trab alhos técnicos dos órgãos de gestão da saúde de vários locais, por meio da montagem de um banco de dados geo gráfico incisivo, apontar com maior precisão os locais relacionais, portanto, com maior probabilidade de focos do Aedes aegypti. Tais áreas necessitariam de maior atenção para eliminação dos criadouros do mosq uito, objetivando a menor proliferação, dispersão e contaminação de pessoas.

\section{MATERIAIS EMÉTODOS}

\section{Área de estudo}

Localizada ao Norte do Estado de Minas Gerais, porção centro-leste do município, a cidade de Montes Claros é composta atualmente por área equivalente a $92.000 \mathrm{~km}^{2}$. A cidade apresenta cento e trinta e seis bairros (136), de acordo com a base cartográfica (Figura 1) fornecida pela Secretaria Municipal de Planejamento e Coordenação (SEPLAN, 2009). A população estimada para o ano de 2017 é de 402.027 pessoas; a densidade demográfica é superior a $101,41 \mathrm{hab} / \mathrm{km}^{2}$, portanto, é bem povoada. A renda média do trabalhador é de 2,2 salários mínimos, correspondente a $26 \%$ da população que se encontra ocupada; o Índice de Desenvolvimento Humano (IDH) é de 0,77 - o décimo oitavo (18º) de Minas Gerais (IBGE, 2017).

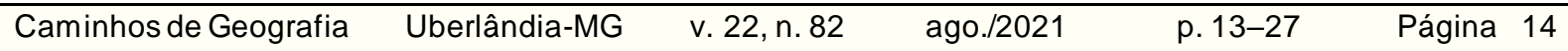


Figura1 - Bairros de Montes Claros e sua localização.

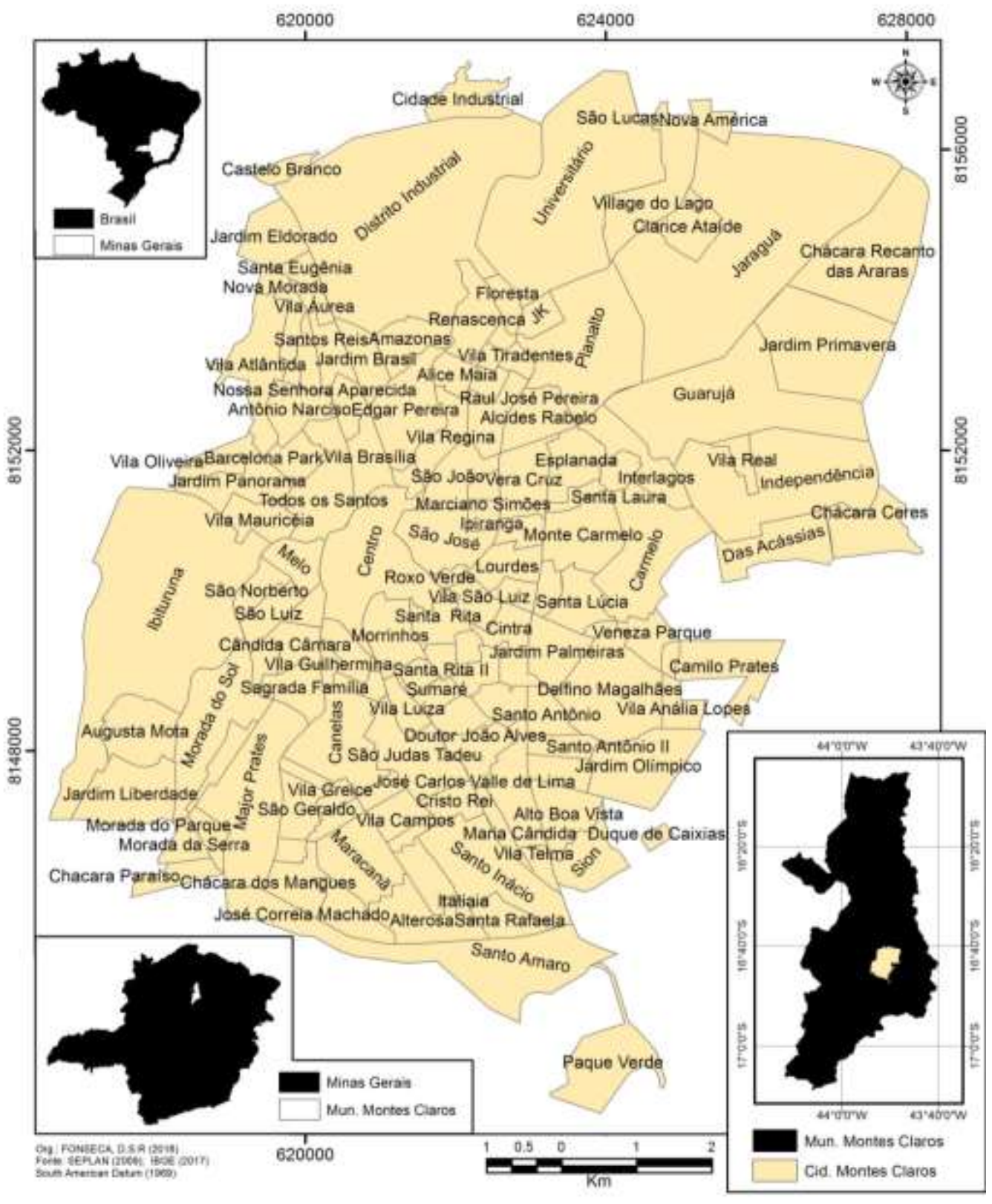

Fonte - SEPLAN (2009); IBGE (2017). Org.: do autor, 2021.

\section{Base de dados}

Os dados para realização do trabalho foram adquiridos junto ao Centro de Controle de Zoonoses (CCZ, 2017), os quais se referem ao Índice Breteau dos bairros da cidade de Montes Claros, período de 2015 a 2017, sendo os dados usados neste trabalho, conforme aquilo que foi fornecido, coletados nos mes es de janeiro, março e outubro. Tal índice tem sido usado como referência para os diagnósticos de inf estação larvária pelo mosquito Aedes aegypti e sua mensuração é feita por meio de uma amostra probabilística dos imóveis ( $10 \%$ de cada bairro) da área urbana. O delineamento amostral é feito por conglomerados, levando em consideração o quarteirão como unidade primária e o imóvel como unidade secundária (MS, 2013). O Índice Breteau leva em conta a relação entre o número de recipientes positivos e o número de imóveis pesquisados, como mostra a seguinte equação:

$$
I B=\frac{\text { recipientes positivos }}{\text { imóveis pesquisados }} * 100
$$


A base cartográfica, com o perímetro dos bairros de Montes Claros, foi adquirida junto à Secretaria de Planejamento (SEPLAN, 2009) em formato vetorial Drawing database (DWG). Para manipular dados relativos à altitude e declividade foi adquirido o modelo digital de elevação Topodata (folha 16S45_ZN), o qual possui resolução espacial aproximada de 30 metros, junto ao Instituto Nacional de Pesquisas Espaciais (INPE, 2018).

\section{Procedimentos operacionais}

A primeira e mais árdua etapa do trabalho consistiu na organização dos dados cedidos pelo CCZ (2017). A base cartográfica, em formato DWG, foi convertida para o formato shapefile, por meio do aplicativo computacional ArcGIS, versão 10.2, e cada perímetro dos bairros foi alterado para polígono, a fim de transformar o arquivo em um banco de dados que pudesse ser passível de inserção dos atributos (Índice Breteau) em sua respectiva tabela. Etapa esta que prosseguiu colocando-se os valores, referentes aos determinados bairros, em correspondência à representação da poligonal criada, e, em seguida, geraram se mapas coropléticos com classes impostas pelo Natural Breaks.

Utilizando a extensão Spatial Statistic Tools, foram realizados os mapeamentos, por ano, dos clusters, com uso do Îndice de Moran (CAMPOS e MACEDO, 2014). Pelo método empregou-se a banda com distância fixa em 5.000 metros. Após a geração dos clusters relativos ao Índice de Moran dos específicos anos, providenciou-se uma análise multicritério, pela qual os mapas foram reclassific ados (extensão do Spatial Analyst Tools - reclassify), impondo-lhes pesos pelo método mencionado, sendo as tais: high-high (peso 100); high-low (peso 80); low-high (peso 40); e low-low (peso 20). Seguidamente a ess a etapa, foi extraída a média ponderada conforme o valor dos pixels (raster calculator) reclassificados, tendo co mo resultado regiões com interação por vizinhança reclassificadas e sintetizadas, as quais foram recorrentes ao longo do período analisado (2015-2017). A montagem deste modelo, o qual se justifica na tentativa de entendimento do problema e na possível realização das decisões, é resumido na álgebra de mapas estruturada conforme orienta Anselin (1999):

$$
A_{i j}=\sum_{K=1}^{n}\left(P_{k} * N_{k}\right)
$$

Onde:

$A_{i j}=$ É a posição do pixelno mapa para análise da matriz;

$n=$ quantidade de mapas (camadas) e variáveis combinadas;

$P_{k}=$ peso atribuído para a camada da variável "k" no conjunto;

$N_{k}=$ valores designados conforme o nível de importância dos componentes nas legendas (variável "k") e com a finalidade do produto.

Os dados sobre altitude e declividade foram manipulados a partir do Modelo Digital de Elevação (MDE) Topodata adquirido, pelo qual se gerou curvas de nível com equidistância de 20 metros, e sucessivamente criaram-se Redes Triangulares Irregulares (TIN). Manipulando esse arquivo, foram obtidos: a declividade média e o desvio-padrão; a altitude mínima, a altitude máxima, a altitude média e o desvio-padrão da elevação de cada bairro, método similar ao descrito em Silva e Rodrigues (2009). Baseado no mesmo MDE realizou-se o mapeamento dos cursos hídricos, os quais tiveram como parâmetro a direção de fluxo das águas (PELUZIO et al., 2010).

A partir da disposição dos valores mínimos, máximos, médios, e, os desvios padrão relativos aos modelos digitais das superfícies dos bairros (clusters), todos os dados gerados foram salvos em tabela digital para realização dos testes estatísticos, no software Bioestat, versão 5.0 (AYRES, 2007), pelo qual, com todos os bairros, foi feita a análise descritiva, teste de hipóteses - Anova seguida pelo teste de Tukey.

Logo após, tendo os clusters como áreas amostrais, a princípio foram testadas todas as variáveis pelo método Stepwise progressivo, o qual providenciou a mais acertada eliminação dos fatores que enf raqueceram o poder explicativo do coeficiente de determinação $\left(R^{2}\right)$. Realizou-se o teste de valores extremos, com base nos desvios, e tais outliers foram eliminados. Finalmente, houve exames de regressão linear múltipla da infestação do mosquito Aedes aegypti em associação com as variáveis sobre elevação do terreno. A regressão linear múltipla é indicada quando a análise exig e modelos com maior complexidade em relação ao desempenho associativo em apenas duas variáveis (AYRES, 2007; BRACAENSE e GUIMARÃES, 2008; ALMEIDA et al., 2009; MARTINS, 2010).

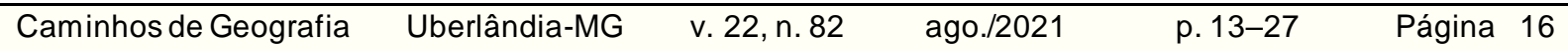




\section{RESULTADOS E DISCUSSÃO}

Os dados referentes ao Índice Breteau sobre a infestação larvária pelo mosquito Aedes aegypti nos bairros da cidade Montes Claros, anos 2017, 2016 e 2015, apresentaram as correspondentes médias: 5,4, 4,5 e 4,3\% - sendo o ano 2017 e o 2015 , significativamente diferentes ( $<<0.05$ ), e a relação entre os demais consideradas iguais, conforme o teste de hipóteses empregado. Os valores apresentaram elevada variabilidade, aqueles com maior dispersão são os do ano 2015, coeficiente de variação igual a 98,4\%; e os de menor dispersão são os do ano 2016, coeficiente de variação igual a 65,1\%; tal como pode ser verificado na Tabela 1.

Tabela 1 - Estatística descritiva dos dados referentes ao Índice Breteau dos anos 2017, 2016e 2015, sobre infestação larvária pelo mosquito Aedesaegyptina cidade Montes Claros-MG.

\begin{tabular}{lccc}
\hline & $\mathbf{2 0 1 7}$ & $\mathbf{2 0 1 6}$ & $\mathbf{2 0 1 5}$ \\
\hline Amostra & 136 & 136 & 136 \\
Valor mínimo & 0 & 0 & 0 \\
Valor máximo & 18.5 & 15.5 & 29.5 \\
Mediana & 4.9 & 4.2 & 3.3 \\
10 quartil & 3.0 & 2.7 & 1.7 \\
3o quartil & 7.1 & 6.0 & 6.4 \\
Média & 5.4 & 4.5 & 4.3 \\
Desvio-padrão & 3.7 & 2.9 & 4.2 \\
Coef. Var. (\%) & 68.6 & 65.1 & 98.4 \\
\hline
\end{tabular}

Fonte - dados do CCZ(2017). Org.: do autor, 2018.

Para o ano de 2015, os bairros com Índice Breteau mais alto, 12,8\%, foram: Sion, região leste; e São Luiz, região central. Outros locais com valores superiores a 7,1\% foram vistos em locais como: Village do Lago, região norte; Vila Anália Lopes, região leste; e Melo, posicionado na região central. O ano 2016 apresentou valores acima de 9,1\% em bairros como: Nova América, região norte; Monte Carmelo, reg ião leste; e o bairro Nossa Senhora das Graças, região sul. Valores acima de 6,1\% foram vistos, por exemplo, no bairro Village do Lago; Vila Anália Lopes; e no lbituruna, região oeste. Em 2017 foram no tadas áreas com valores superiores a 11,7\% do índice de infestação larvária em bairros como: Clarice Ataíde, reg ião norte; Santa Rita Il e Sumaré, na região leste da cidade. Áreas com o índice superior a 7,1\% estiveram nos seguintes locais: Floresta, região norte; Independência, região leste; Sion e no lbituruna.

No recorte de tempo deste estudo nota-se, por meio da Figura 2, que a precipitação da região de Mo ntes Claros é concentrada entre os meses de outubro a março, tendo sido 2015 o ano com as chuvas mais concentradas no mês de dezembro (superior a $450 \mathrm{~mm}$ ); o ano 2016 foi aquele com a melhor distribuição; e 2017 apresentou menor oferta pluviométrica. As temperaturas têm suas médias diminuídas em torno de $3^{\circ} \mathrm{C}$, em média, entre os meses de maio e agosto; e há déf icit hídrico em tais meses (INMET, 2018).

Figura 2 - Precipitação total $(\mathrm{mm})$ e tem peratura máxima $\left({ }^{\circ} \mathrm{C}\right)$ no período entre 2015 e 2017 na cidade de Montes Claros-MG.

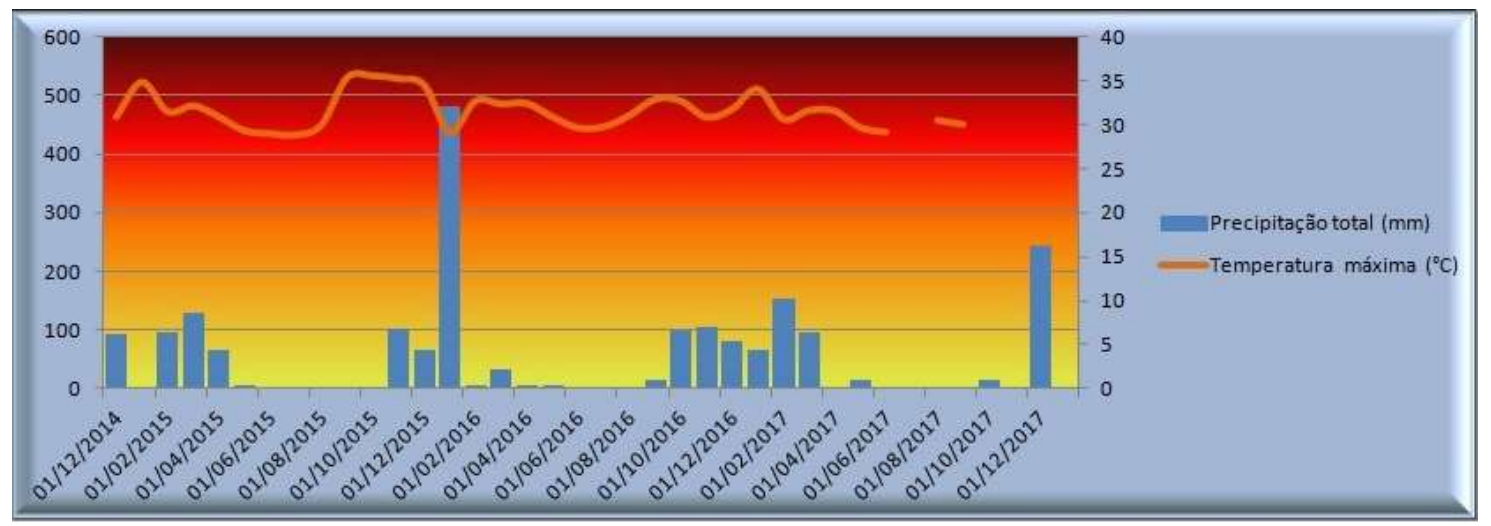

Fonte - INMET (2018). Org.: do autor, 2018.

$\begin{array}{lll}\text { Caminhos de Geografia } \quad \text { Uberlândia-MG } & \text { v. 22, n. } 82 \quad \text { ago./2021 }\end{array}$

p. 13-27

Página 17 
O comportamento da infestação larvária pelo mosquito Aedes aegypti em consequência da relação pluviosidade e capacidade de escoamento leva ao estabelecimento de conexões entre dados sobre elevação do terreno e incidência larvária do mosquito. Nesse sentido, é compreendido que a cidade, Montes Claros, apresenta altitude média de 660 metros, sobretudo na região central. As áreas de maior elevação $(900 \mathrm{~m})$ estão situadas na direção Sul (sudeste) e as de menor $(600 \mathrm{~m})$ localizam -se ao Norte (noroeste). O terreno, em geral, é pouco abrupto (declividade média em torno de $2,35^{\circ}$ ), áreas com maior desnível estão posicionadas a Oeste e Sul da área urbana, tal como pode ser interp retado por meio da Figura 4. Essa análise se torna necessária porque as diferenças no nível do relevo montesclarence poderiam interferir no comportamento da infestação relativa ao inseto em estudo, tendo em vista, por exemplo, a velocidade de escoamento das águas, sua distribuição e acumulação ao longo do perímetro urbano.

Figura 4 - Intervalos de classesaltimétricas e curvas de nível representando a morfologia do relevo da cidade Montes Claros-MG.

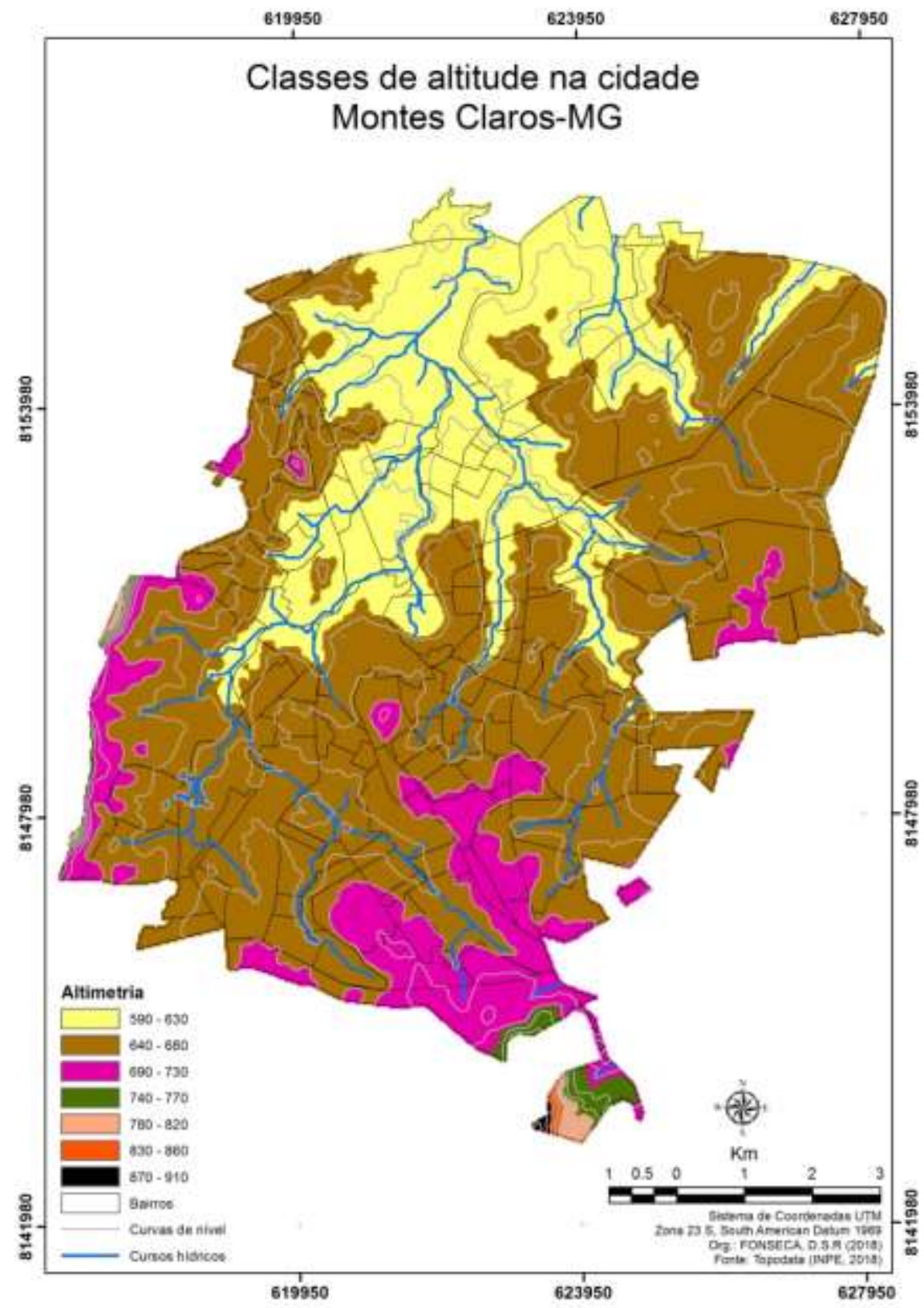

Fonte - SEPLAN (2009); INPE (2018). Org.: do autor, 2018.

Em busca de uma análise mais apurada do ambiente urbano em questão, procedeu-se com a identificação dos agrupamentos de aglomerados espacialmente autocorrelacionad os na cidade, ao ser 
aplicado o Índice de Moran nas taxas do Breteau como critério técnico-científico (ANSELIN, 2005). A Figura 5 apresenta a utilização desse recurso, pela qual é verificada a maior concentração dos clusters (classe High-High) sobretudo em torno dos bairros: Ipiranga, Marciano Simões e Edgar Pereira (consulte a Figura 1, se necessário). Estas áreas foram hierarquicamente acompanhadas, em segundo plano, daquelas em torno do bairro independência (classe High-Low). Os clusters das áreas com muito baixa taxa (classe Low-Low) estão, em geral, ao sul do perímetro urbano, ocorrendo nesses espaços a autocorrelação negativa dos focos da infestação larvária, tratando-se daqueles em torno dos bairros: Santo Amaro e Chácaras Paraíso. O Índice Global de Moran ficou superior a 0,70 nos três anos mostrados, indicando alta correlação espacial por vizinhança (CAMPOS e MACEDO, 2014).

Figura 5- Mapeamento de clusters, pelo Índice de Moran, mostrando os aglomerados espacialmente autocorrelacionados no decorrer do tempo, pelo Índice Breteau do Aedes aegypti, na cidade Montes Claros-MG.

\section{Indice de Moran ao longo dos anos (2015 a 2017)
na cidade: Montes Claros-MG}

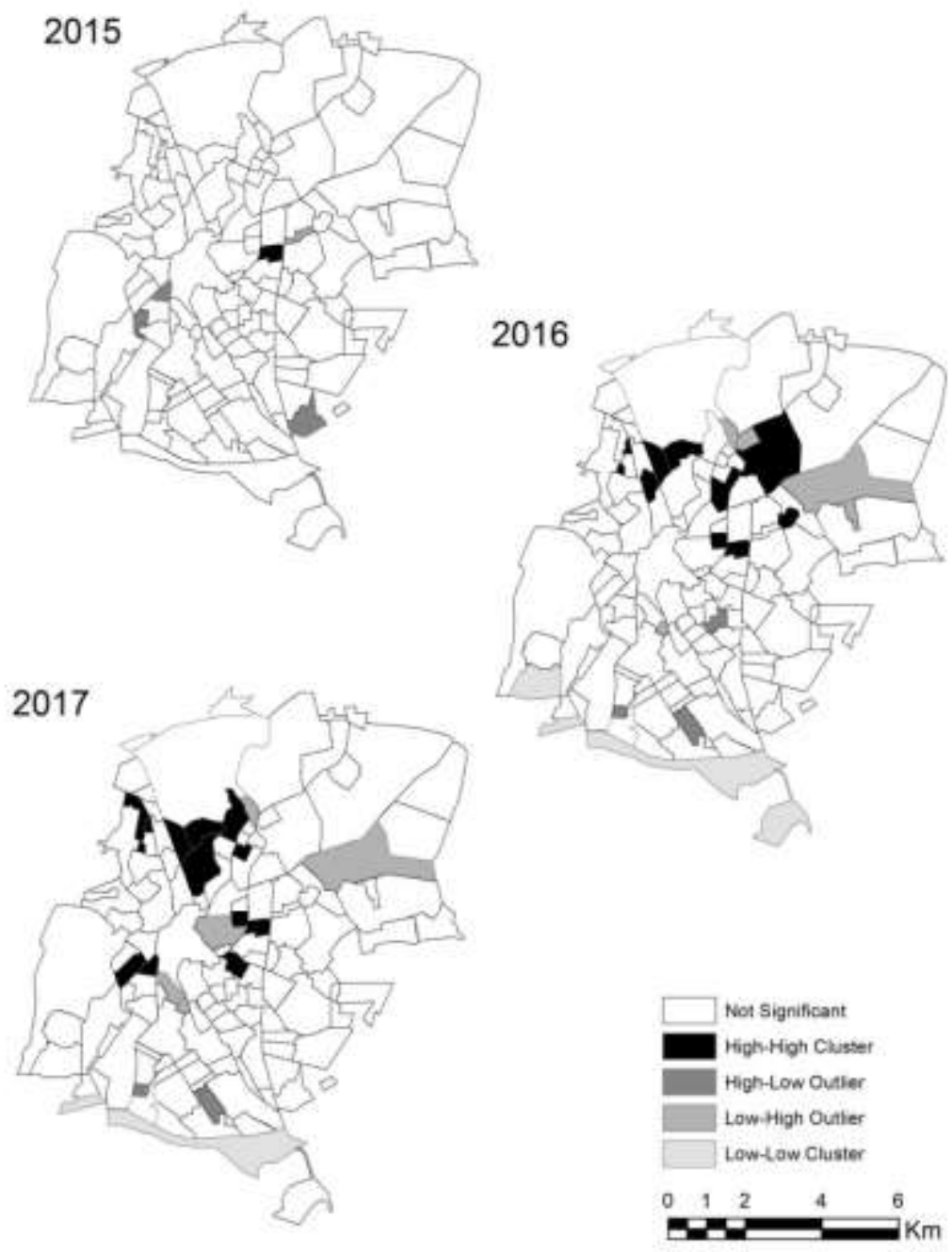

Fonte - SEPLAN (2009); CCZ(2017). Org.: do autor, 2018.

Diante da possibilidade metodológica de tornar a inf ormação cartográfica mais sintética e, portanto, mais incisiva para interpretação das áreas críticas com maior ocorrência de focos do mosquito Aedes aegypti, providenciou-se a análise temporal multicritério (ANSELIN, 1999). Por meio da Figura 6 são observados os clusters dos locais com muito alta (vermelho-intenso) autocorrelação espacial, bairro Ipiranga; e os de

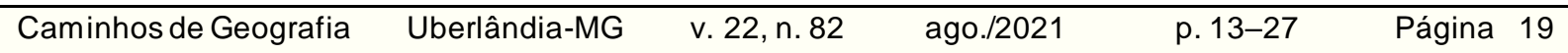


muito baixa autocorrelação espacial (amarelo-pastel), nas adjacências do bairro Santo Amaro. Estes bairros influenciam a infestação larvária do $A$. aegypti, nas regiões ao redor, para taxas mais elevadas (muito-alta) e para taxas reduzidas (muito baixa).

Figura 6 - Mapa sintético do Índice de Moran, dos anos 2015, 2016 e 2017, provenientes do Índice Breteau, relativo ao mosquito Aedesaegypti, na cidade Montes Claros-MG.

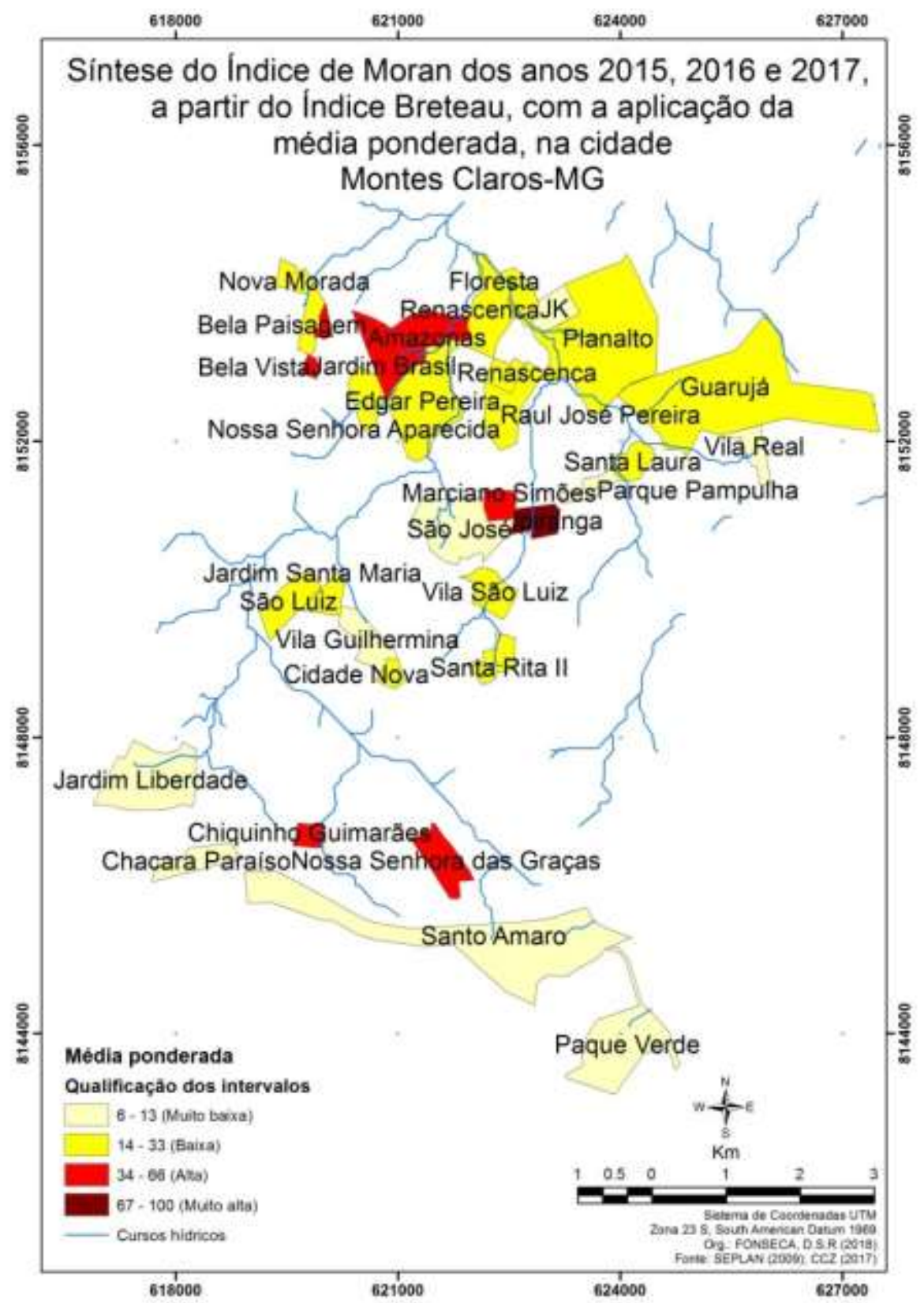

Fonte - SEPLAN (2009); CCZ(2017). Org.: do autor, 2018.

Os locais delimitados foram tomados como área de pesquisa, com a inserção de variáveis es paciais, tal como mostra a Figura 7. A partir desse modelo, restringiu-se o universo de cento e trinta e seis (136) bairros para trinta e um (31) locais amostrais, sendo estes os mais representativos do fenômeno em análise (ANSELIN, 2005; CAMPOS e MACEDO, 2014). Além da delimitação, pode ser notado que os bairros estão em posições geográficas diversificadas ao longo do perímetro urbano, assim como a declividade parcial dos seus terrenos, enquanto as altitudes variaram entre os 620 aos 760 metros. 
Figura 7 - Principais dados adquiridos, gerados e manipulados, sob a área amostral (Índice de Moran), relativaà infestação larvária do Aedes aeypti em Montes Claros-MG.

\begin{tabular}{|c|c|c|c|c|c|c|c|}
\hline Bairro & Regiāo & Bret. 2017 & Bret. 2016 & Bret.2015 & Decliv. Méd & Altit. Med & Altit. Max \\
\hline Amazonas & Norte & 15.4 & 9.1 & 2.4 & 2.5 & 626.0 & 640.0 \\
\hline Bela Paisagem & Oeste & 15.6 & 15.6 & 7,3 & 0.0 & 660.0 & 660.0 \\
\hline Bela Vista & Oeste & 15.8 & 9.8 & 9.1 & 11.3 & 669.0 & 680.0 \\
\hline Chacara Paraiso & Sul & 0.0 & 0.0 & 0.0 & 0.0 & 660.0 & 660.0 \\
\hline Chiquinho Guima & Sul & 18.5 & 8.7 & 3.9 & 0.0 & 660.0 & 660.0 \\
\hline Cidade Nova: & Sut & 0.0 & 0.0 & 107 & 0.0 & 670.0 & 670.0 \\
\hline Edgar Pereira & Central & 14.0 & 6.0 & 7.1 & 0.0 & 620.0 & 620.0 \\
\hline Floresta & Norte & 0.0 & 0.0 & 4.8 & 0.0 & 620.0 & 620.0 \\
\hline Guaruja & Leste & 0.0 & 0.0 & 0.0 & 31.0 & 656.0 & 660.0 \\
\hline Ipiranga & Leste & 9.8 & 9.8 & 10.1 & 0.0 & 640.0 & 640.0 \\
\hline Jardim Erasil & Norte & 9.8 & 7.7 & 1.1 & 2.3 & 6310 & 640.0 \\
\hline Jardim Liberdad & Oeste & 0.0 & 0,0 & 12.5 & 2.2 & 668.0 & 680.0 \\
\hline Jardim Santa Ma & Central & 11.7 & 7.1 & 25.5 & 0.0 & 640.0 & 640.0 \\
\hline JK: & Norte & 3.0 & 2.5 & 3.5 & 0.1 & 620.0 & 620.0 \\
\hline Marciano Simdes & Leste & 10.1 & 10.1 & 9.0 & 0.0 & 640.0 & 640.0 \\
\hline Nossa Senhora A. & Norte & 8.3 & 8.3 & 7.2 & 0.2 & 630.0 & 630.0 \\
\hline Nossa Senhora d & Sul & 14.6 & 14.6 & 1.9 & 0.0 & 680.0 & 680.0 \\
\hline Nova Morada & Oeste & 8.5 & 6.2 & 0.0 & 6.1 & 650.0 & 680.0 \\
\hline Paque Verde & sul & 0.0 & 0.0 & 0.0 & 7.6 & 760.0 & 900.0 \\
\hline Parque Pampulha & Leste & 5.2 & 3,8 & 0.0 & 5.2 & 633.0 & 640.0 \\
\hline Planalto & Norte & 7,0 & 7.0 & 8.1 & 1.1 & 633.0 & 640.0 \\
\hline Raul Josè Perei & Norte & 7.4 & 7.4 & 1.5 & 0.0 & 640.0 & 640.0 \\
\hline Renascenca & Norte & 8.3 & 6.5 & 4.2 & 0.0 & 620.0 & 620.0 \\
\hline Santa Laura & Leste & 7.9 & 7.9 & 7.3 & 0.0 & 630.0 & 630,0 \\
\hline Santa Rita H & Leste & 13.7 & 13.7 & 10.6 & 0.0 & 660.0 & 660.0 \\
\hline Santo Amaro & Sul & 0.0 & 0.0 & 0.0 & 3.4 & 699.0 & 760.0 \\
\hline Săo José & Central & 1.7 & 1.7 & 3.5 & 0.0 & 636.0 & 652.0 \\
\hline 5ăo Luit & Central & 10.0 & 6.2 & 8.7 & 0.0 & 646.0 & 655.0 \\
\hline Vila Guithermin & Central & 1.0 & 1.0 & 1.2 & 0.0 & 661.0 & 676.0 \\
\hline Vila Real & Leste & 1.6 & 1.6 & 5.9 & 0.0 & 660.0 & 660.0 \\
\hline Vila Säo Luiz & Leste & 10.0 & 6.2 & 8.7 & 0.0 & 6450 & 654.0 \\
\hline
\end{tabular}

Fonte - CCZ(2017); INPE (2018). Org.: do autor, 2018.

A área em perspectiva apresentou médias para o Índice Breteau, anos 2015, 2016 e 2017, correspondentes a: 7,3; 5,7 e 5,6\%. Os valores demonstram menor variabilidade quando comparados com os dados generalizados de todos os bairros de Montes Claros. Aqueles com maior dispersão são os do ano 2015, coeficiente de variação igual a 94,3\%; e os de menor dispersão são os do ano 2017, coeficiente de variação igual a 79,3\%; tal como pode ser verificado na Tabela 3.

Tabela 3 - Estatística descritiva dos dados referentes aos Índices Breteaures dos anos 2017, 2016 e 2015, sobre infestação larvária pelo m osquito Aedes aegypti, na área am ostral gerada a partir da síntese do Índice de Moran.

\begin{tabular}{lccc}
\hline & $\mathbf{2 0 1 7}$ & $\mathbf{2 0 1 6}$ & $\mathbf{2 0 1 5}$ \\
\hline Amostra & 31 & 31 & 31 \\
Valor mínimo & 0 & 0 & 0 \\
Valor máximo & 18.5 & 15.5 & 25.5 \\
Mediana & 8.2 & 6.2 & 4.7 \\
1quartil & 1.3 & 1.3 & 1.3 \\
3ㅇ quartil & 10.8 & 8.4 & 7.3 \\
Média & 7.3 & 5.7 & 5.6 \\
Desvio padrão & 5.8 & 4.5 & 5.3 \\
Coef. Var. (\%) & 79.3 & 79.7 & 94.3 \\
\hline
\end{tabular}

Fonte - dados do CCZ(2017). Org.: do autor, 2018. 
Tabela 5 - Modelo de regressãolinear múltipla usado para predição da variável dependente, o Índice Breteau do Aedes aegypti ano2017, com as variáveis independentes: Índice Breteau do Aedes aegypti dosanos2016e 2015; a elevação média e a altitude máxima dos bairros.

\begin{tabular}{llccc}
\hline & \multicolumn{1}{c}{$\mathbf{R}$} & \multicolumn{1}{c}{$\mathbf{R}^{\mathbf{2}}$} & $\mathbf{R}^{\mathbf{2}}$ ajustado & P valor \\
& \multicolumn{1}{c}{0.986} & 0.973 & 0.96 & 0.0001 \\
\hline $\begin{array}{l}\text { Variável } \\
\text { dependente }\end{array}$ & & Coeficiente & $\mathbf{t}$ & $\mathbf{p}$ \\
\hline \multirow{3}{*}{$\begin{array}{l}\text { Índice Breteau } \\
2017\end{array}$} & Interseção & 20.241 & 2.200 & 0.038 \\
\cline { 2 - 5 } & $\begin{array}{l}\text { Variáveis } \\
\text { independentes }\end{array}$ & & & \\
\cline { 2 - 5 } & Breteau 2016 & 1.046 & 23.94 & 0.0001 \\
\cline { 2 - 5 } & Breteau 2015 & 0.023 & 0.541 & 0.593 \\
\cline { 2 - 5 } & Alt. Méd. & -6.460 & -2.157 & 0.041 \\
\cline { 2 - 5 } & Alt. Máx. & 3.317 & 1.847 & 0.077 \\
\hline
\end{tabular}

Fonte - CCZ(2017); INPE (2018). Org.: do autor, 2018.

Há, portanto, conforme os resultados mostrados na Tabela 5, forte correspondência associativa entre as taxas de infestação nos bairros, relativas ao ano anterior (2016) e o seguinte (2017), e, a partir daí, o desencadeamento da predisposição à maior suscetibilidade dos focos larvários, tendo como pressupos to as características do ambiente físico.

Em confluência com os resultados alcançados, qual a relação da dinâmica espacial poderia explicar o evento mencionado? Se as médias de altitude em determinados bairros são mais baixas em relação ao conjunto, então maiores são as possibilidades para se encontrar focos do mosquito Aedes aegypti em tais lugares. Mas, ao mesmo tempo, essa probabilidade pode ser agravada à medida que a elevação máxima do terreno for superior. Esses fatores estão relacionados às características de certos locais que foram pavimentados, sendo eles, por exemplo, antigas várzeas e os campos alagadiços, os quais estão nas altitudes mais baixas dos terrenos. Em tais pontos geográficos falados é mais propício o acúmulo de água parada, tal como mostra a Figura 9.

Figura 9 - Esquema ilustrativo pelo qual pode ser interpretada a dinâmica da água, parada nos locais de menor e maior e elevação do terreno, e o movimento hídrico no declive.

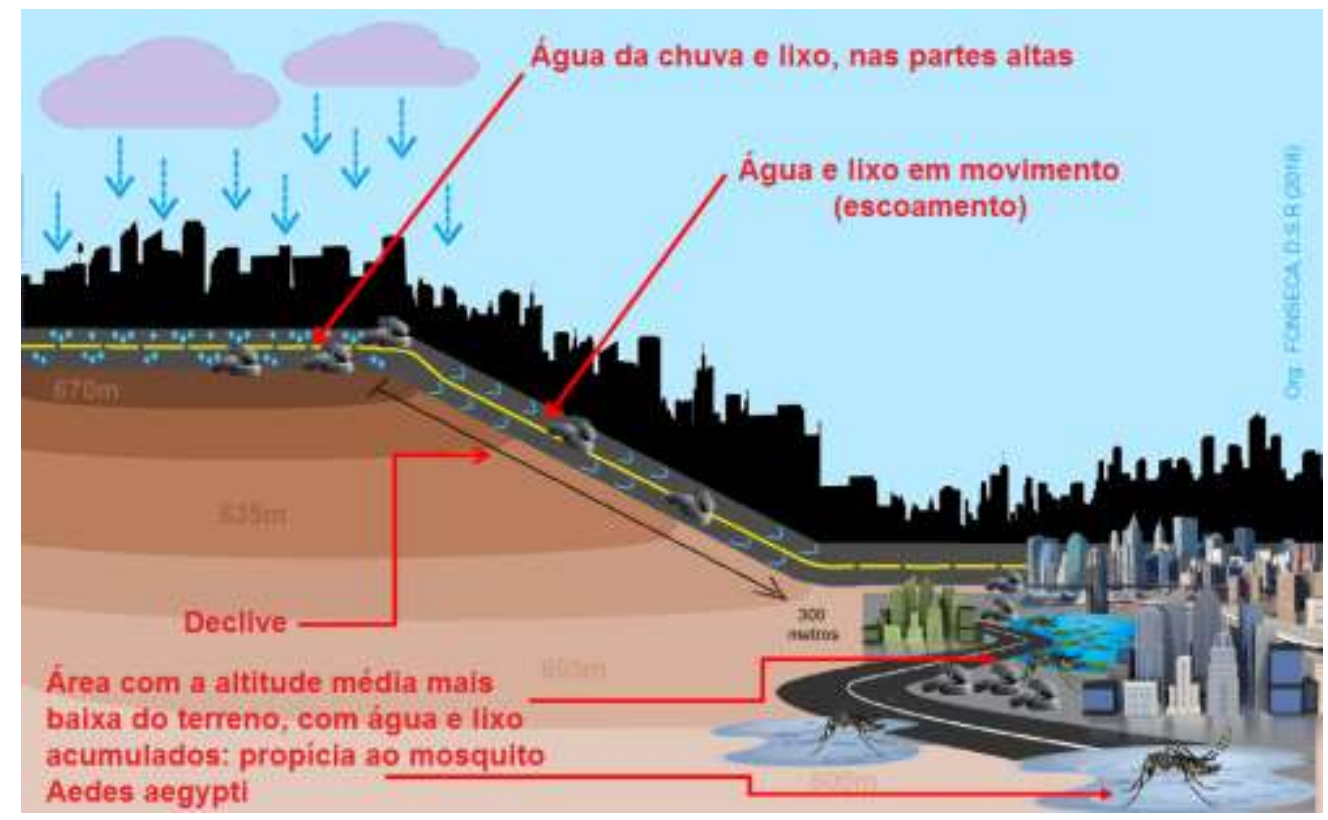

Fonte - Elaborado pelo autor, 2018.

$\begin{array}{llll}\text { Caminhos de Geografia } & \text { Uberlândia-MG } & \text { v. 22, n. } 82 & \text { ago./202 }\end{array}$

p. 13-27

Página 23 
É necessário ser salientado que o foco deste estudo é a análise dos ambientes externos às edificações, e dessa forma, busca-se praticar a geografia da saúde sob o ponto de vista não convencional daqueles propagados pelos meios de comunicação. Para acrescentar, é sabido que atualmente inexiste vacina eficaz a todos os sorotipos circulantes transmitidos pelo Aedes aegypti, tornando a melhor opção o controle dessa praga, a qual está respaldada numa ação combinada de fatores como: o ser humano, o vírus, o vetor e o ambiente (JENTES et al., 2011; RIZZI et al., 2017). Nessas perspectivas, é necessário melhorar o escoamento superficial das águas nas zonas críticas onde há maior ocorrência desse vetor de doenças.

Ao tomar como parâmetro as premissas descritas, torna-se imprescindível a busca da mel hor qualidade urbana, com a diminuição dos ambientes insalubres, tais como os depósitos de entulho, lugares altamente impermeabilizados, com bueiros entupidos ou a ausência destes. Assim sendo, o planejamento territorial da cidade, por meio do plano diretor, deve levar em consideração a manutenção dos espaços com vegetação natural e aperfeiçoar o saneamento básico citadino, pois quando há acúmulo de resíduos sólidos em áreas não controladas, maior é a proliferação do mosquito $A$. aegyptinas ép ocas com maior pluviosidade (ALMEIDA e SILVA, 2018).

Essa relação entre a maior of erta pluviométrica e o aumento das taxas de ocorrência larvária pôde ser comprovada com a análise entre a Figura 2 e o teste de Tukey realizado. Através destes, é notada a maior ocorrência e distribuição das chuvas na primavera-verão do ano 2016, e no período posterio r, em 2017, houve o episódio dos focos do mosquito de forma significativamente igual ao ano anterior. Tal como os índices de precipitação desse período, há na literatura abordagens mostrando picos de incidência do Aedes aegypti, e de pessoas doentes por vírus transmitidos por esse vetor, os quais acontecem, sobretudo, após épocas de chuva com totais acumulados superiores a $100 \mathrm{~mm}$ (FERREIRA et al., 2018; LIMA et al., 2018).

Em épocas de chuva o escoamento superficial montesclarense é ineficaz. Várias ruas ficam alagadas e o asfalto se deteriora. Buracos aparecem semestralmente nos mesmos locais e permanecem por tempo indeterminado. Até que comece a operação "tapa buracos" da prefeitura, tais imperfeições nas vias podem funcionar como criadouros da larva, a qual se torna o mosquito transmissor da febre amarela e do zika vírus (LANA et al., 2018).

O entulho arrastado a partir das áreas mais altas para as mais baixas pelo escoamento das águas, é outro fator que pode agravar a disseminação do Aedes, porque o lixo acumulado nos bairros com altitudes médias mais baixas vira habitat do inseto. Existem campanhas para que a população reaja na contenção desse vetor de doenças e ameaça à saúde social. Mas essas ações mascaram a culpa dos gesto res públicos e dos planejadores, os quais mantêm e criam espaços ineficientes, colocando to da a cu lpa da proliferação das doenças originadas através do Aedes sobre a população desinformada, a qual supostamente, não higieniza adequadamente seus ambientes.

Estudos têm mostrado a plasticidade do mosquito $A$. aegypti, o qual na ausência de criadouros artificiais para depósito de seus ovos, a fêmea do inseto se adapta migrando-se para criadouros naturais, tais como em bromélias e cascas de árvores. A escassez da pluviosidade pode acarretar a adaptação do inseto para lugares de estocagem d'água, ou ainda, recintos subterrâneos, onde ocorre a drenagem da água superficial, mostrando que a vigilância e o controle desse mosquito vetor devem ser realizados constantemente (LIMA-CÂMARA et al., 2016; BERMUDI et al., 2017).

A identificação das regiões potenciais para a prevalência dos focos larvários do mosquito vetor, por meio do Índice Moran, mostrou-se eficiente (CARVALHO et al., 2008). A partir desses espaços foi possível compreender algumas características da ambiência física, relativos à altitude e a declividade, as quais são preponderantes para a inf estação. Nesses lugares identificados a vigilância em saúde deve ser prioritária, através de ações estratégicas para controle, que deveriam começar "debaixo para cima" - nas altitudes mais baixas até as superfícies mais elevadas dos bairros afetados.

O tema é complexo e os riscos aos quais as populações estão submetidas são vários, pois se trata da praga transmissora da dengue; da febre amarela (JENTES et al., 2011); da febre chikungunya (CHAVES et al., 2012) e do zika vírus (CAMPOS et al., 2015) - o mosquito Aedes aegypti. Cabe aos gestores públicos se comprometerem mais no planejamento e manutenção das cidades, também, s ob o ponto de vista epidemiológico. É necessário que se acabe com o discurso unilateral no qual é apontada a população, sobretudo os mais desprovidos de renda, como os únicos culpados pela dis seminação de doenças pela falta de higiene. Aos planejadores urbanos, cabe a eles reverterem a má fama que adquiriram em projetos de cidades as quais não deram certo em diversos aspectos, a não ser, por exemplo, na segregação e no aprisionamento das pessoas residentes (CARLOS, 2018). 


\section{CONCLUSÕES}

O coeficiente de determinação $\left(R^{2}\right)$ final ficou em 0,973 - o que denota alto poder preditivo, para a combinação das variáveis independentes trabalhadas. O Índice Breteau de 2016 é aquele com maior peso explicativo $(p<0,01)$ entre o conjunto de dados para a explicação da infestação larvária ocorrida em 2017. É verificado que a altitude média foi significativa nessa associação $(p<0,05)$, e apresentou correlação negativa conforme o coeficiente parcial de regressão $(-6,460$ e $t=-2,157)$. Em segundo plano, a altitude máxima foi significativa $(p<0,08)$. Dessa forma, ocorre a correlação associativa entre o período anterior e o seguinte, e nos locais onde houve maior incidência larvária, quanto mais baixa é a elevação média, maior é a infestação pelo mosquito, devido a maior propensão ao acúmulo de água parada.

Os fatores mostrados estão relacionados à maior capacidade de certos locais para acomodação da água, entre estes, por exemplo, antigas várzeas e os campos alagadiços, posicionados nas baixas altitudes dos terrenos, levando à maior proliferação larvária do Aedes aegypti. Nestes, é mais propício o acúmulo de água parada vindo das partes altas, em comparação aos locais com pronunciada declividade. Portanto, é necessário que sejam realizadas mudanças na estrutura das cidades, caso o objetivo seja realmente minimizar a crise urbana e promover o bem coletivo.

\section{AGRADECIMENTOS}

Os autores agradecem à Fundação de Amparo à Pesquisa de Minas Gerais (FAPEMIG) pelo apoio e financiamento desta pesquisa, por meio da concessão da bolsa de doutorado. Gratidão prolongada ao Programa de Pós-Graduação em Geografia, do Instituto de Geociências (IGC), da Universidade Federal de Minas Gerais (UFMG).

\section{REFERÊNCIAS}

ALMEIDA, A.S; MEDRONHO, R.A.; VALÊNCIA, L.I.O. Análise espacial da dengue e o contexto socioeconômico no município do Rio de Janeiro, RJ. Revista de Saúde Pública, v. 43, n. 4, p. 666 -673, 2009. https://doi.org/10.1590/S0034-89102009000400013

ALMEIDA, C, A. P.; SILVA, R. M. Análise da ocorrência dos casos de dengue e sua relação com as condições socioambientais em espaços urbanos: os casos de João Pessoa, Cabedelo e Bayeux, no Estado da Paraíba - Brasil. Hyg eia, n. 14, v. 27, p. 56-79, 2018.

ANSELIN, L. Exploring Spatial Data with GeoDaTM: A Workbook. University of Illinois: Urbana, 2005.

ANSELIN, L. Interactive techniques and exploratory spatial data analysis. In.: LONGLEY, P; GOODCHILD, M; MAGUIRE, D.; RHIND, D. (eds.). Geographical Information Systems: principles, techniques, management and applications. New York: Jonh Wiley \& Sons, 1999.

AYRES, M. BioEstat. Versão 5.0. Soc. Civ. Mamirauá, MCT - CNPq. Belém: 2007, 290p.

BERMUDI, P.M.M; KOWALSKI, F; MENZATO, M.M; FERREIRA, M.C; PASSOS, W.B.S.; OKU, V.J.A. Criadouro de Aedes aegypti em reservatório subterrâneo de água da chuva: um alerta. Revista de Saúde Pública, v. 51, n. 122, p. 1-5, 2017. https://doi.org/10.11606/S1518-8787.2017051000087

BRACARENSE, P.A.; GUIMARÃES, U.V. Métodos Quantitativos Aplicados a Negócios. Curitiba: IESDE Brasil S.A, 2008, 324p.

CAMPOS, M. B.; MACEDO, D.R. Agrupamentos de emigração internacional no Brasil: o papel das red es sociais na formação dos espaços de emigração. Geografia, v. 39, n. 2, p. 257-272, 2014.

CARLOS, A. F. A (org.). Crise urbana. São Paulo: Contexto, 2018, 191p.

CARVALHO, F.M.V; FERREIRA, L.G; LOBO, F.C; DINIZ-FILHO, J.A.F.; BINI, L.M. Padrões de autocorrelação espacial de índices de vegetação MODIS no bioma Cerrado. Revista Árvore, v. 32, n.2, p.279-290, 2008. https://doi.org/10.1590/S0100-67622008000200011

CCZ, CENTRO DE CONTROLE DE ZOONOZES. Prefeitura Municipal de Montes Claros. Divisão de epidemiologia. Guia prático do LIRAa (2011-2017). Montes Claros, 2017.

CONSOLI, R. A. G. B.; OLIVEIRA, R. L. Principais mosquitos de importância sanitária no Brasil. Rio de Janeiro: FioCruz; 1994, 228p. Disponível em: <http://static.scielo.org/scielobooks/th/pdf/consoli9788575412909.pdf>. Acessado em 26 de janeiro de 2018. https://doi.org/10.7476/9788575412909 
FERREIRA, A.C; CHIARAVALLOTI-NETO, F.; MONDINI, A. Dengue em Araraquara, SP: epidemiologia, clima e infestação por Aedes aegypti. Revista de Saúde Pública, v. 52, n. 18, 2018. https://doi.org/10.11606/S1518-8787.2018052000414

FORATINI, O. P. Culicidologia Médica. São Paulo: Edusp, 2002, 862p.

GUPTA B.; REDDY B.P.N. Fight against dengue in India: progresses and challenges. Parasitol Res. doi:10.1007/s00436-013-3342-2, 2013. https://doi.org/10.1007/s00436-013-3342-2

IBGE, INSTITUTO BRASILEIRO DE GEOGRAFIA E ESTATÍSTICA. Cidades (2017). Disponível em: $<$ https://cidades.ibge.gov.br/brasil/mg/montes-claros/panorama>. Acesso: Dezembro de 2017.

INMET, INSTITUTO NACIONAL DE METEOROLOGIA. BDMEP - Banco de Dados Meteorológicos para Ensino e Pesquisa. Disponível em: <http://www.inmet.gov.br/portal/index.php?r=bdmep/bdmep>. Acesso em: Maio de 2018.

INPE, INSTITUTO NACIONAL DE PESQUISAS ESPACIAIS. Modelo TOPODATA (2008). Disponível em: $<$ http://www.webmapit.com.br/inpe/topodata/>. Acesso: Março de 2018.

JENTES, E. S.; POUMEROL, G.; GERSHMAN, M. D.; HILL, D. R.; LEMARCHAND, J.; LEWIS, R. F.; STAPLES, J. E.; TOMORI, O.; SMITH, A. W.; MONATH, T. P. The revised global yellow fever risk map and recommendations for vaccination, 2010: consensus of the Informal WHO Working Group on Geographic Risk for Yellow Fever. The Lancet, v. 11, p. 622-632, 2011. https://doi.org/10.1016/S1473$\underline{3099(11) 70147-5}$

KONISHI E. Issues related to recent dengue vaccine development. Trop Med Health. doi:10.2149/tmh.2011-S0, 2011. https://doi.org/10.2149/tmh.2011-S01

LANA, R.M; MORAIS, M. M; LIMA, T. F. M; CARNEIRO, T. G. S; STOLERMAN, L. M; SANTOS, J. P. C; CORTÊS, J. C; EIRAS, A. E.; CODEÇO, C. T. Assessment of a trap based Aedes aegypti surveillance program using mathematical modeling. PLoS ONE, v. 13, n. 1, p. 1-16, 2018. https://doi.org/10.1371/journal.pone.0190673

LIMA, S.F.S; BARROZO, L.V.; MATAVELI, A.V. Temperatura de superfície e precipitação que influenciam na incidência do Aedes aegypti em São Paulo. Revista do Departamento de Geografia, volume es pecial, p. 174-183, 2018. https://doi.org/10.11606/rdg.v0ispe. 145697

LIMA-CAMARA, T.N; URBINATTI, P.R.; CHIARAVALLOTI-NETO, F. Encontro de Aedes aegypti em criadouro natural de área urbana, São Paulo, SP, Brasil. Revista de Saúde Pública, v. 50, n. 3, p. 1-4, 2016.

MARTINS, G. A. Estatística geral e plicada. São Paulo: Atlas, 2010, 421p.

MESSINA, J. P.; BRADY, O. J.; PIGOTT, D. M.; GOLDING, N.; KRAEMER, M. U. G.; SCOTT, T. W.; WINT, G. R. W.; SMITH, D. L.; HAY, S. I. The many projected futures of dengue. Nature, n. 13, p. 230-239, 2015. https://doi.org/10.1038/nrmicro3430

MORENO, E. S.; BARATA, R. C. B. Methodology for definition of yellow fever priority areas, based on environmental variables and multiple correspondence analyses. PLoS Biology, v. 6, n. 7, p. 1-7, 2012. https://doi.org/10.1371/journal.pntd.0001658

MS, MINISTÉRIO DA SAÚDE. Secretaria de Vigilância em Saúde. Departamento de Vigilância das Doenças Transmissíveis. Levantamento Rápido de Índices para Aedes aegypti (LIRAa) para vigilância entomológica do Aedes aegyptino Brasil: metodologia para avaliação dos índices de Breteau e Predial e tipo de recipientes. Brasília: Ministério da Saúde, 2013. Disponível em: < http://bvsms.saude.gov.br/bvs/publicacoes/manual_liraa_2013.pdf>. Acesso: Janeiro de 2018.

PELUZIO, P.M.O; SANTOS, A.R; FIEDLER, N.C; COELO, A.L.N; EUGENIO, F.C; LOUZADA, F.L.R.O; SAITO, N.S; FERRARI, J.L; QUARTO-JÚNIOR, P.; BATISTA, R.S. Mapeamento de Áreas de Preservação Permanente no ArcGIS 9.3. Alegre: CAUFES, 2010, 58p.

PINHEIRO, F. P.; CORBER, S. J. Global Situation of Dengue and dengue Haemorrhagic fever, and its Emergence in the Americas. World Health Statistics Quarterly Rapport Trimestriel de statistique sanitaires Mondiales, v.50, n.3/4, p. 161-169, 1997.

RIZZI, C. B; RIZZI, R. L; PRAMIU, P. V; HOFFMANN, E.; CODEÇO, C. T. Considerações sobre a dengue e variáveis de importância à infestação por Aedes aegypti. Hygeia, v. 13, n. 24, p. 24-40, 2017.

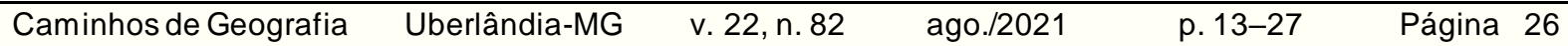


SAWYER, D. O.; LEITE, I. C.; ALEXANDRINO, R. Perfis de utilização de serviços de saúde no Brasil. Ciência \& Saúde Coletiva, v. 7, n. 4, p. 757-776, 2002. https://doi.org/10.1590/S1413$\underline{81232002000400012}$

SEPLAN, SECRETARIA MUNICIPAL DE PLANEJAMENTO E COORDENAÇÃO. Mapa Perímetro Baimos (2009). Disponível em: < http://www.montesclaros.mg.gov.br/infraestrutura/mapas.htm>. Acesso: Março de 2018.

SILVA, T. I.; RODRIGUES, S. C. Tutorial de cartografia geomorfológica ArcGIS 9.2 e ENVI 4.0. Rev ista Geográfica Acadêmica, v.3, n.2, p. 1-66, 2009.

Recebido em:31/05/2020

Aceito para publicação em: 14/06/2021 